\title{
Schwarzes Brett
}

DREILÄNDERKONGRESS

\section{Umfangreiche Fortbildung für psychiatrisch Tätige}

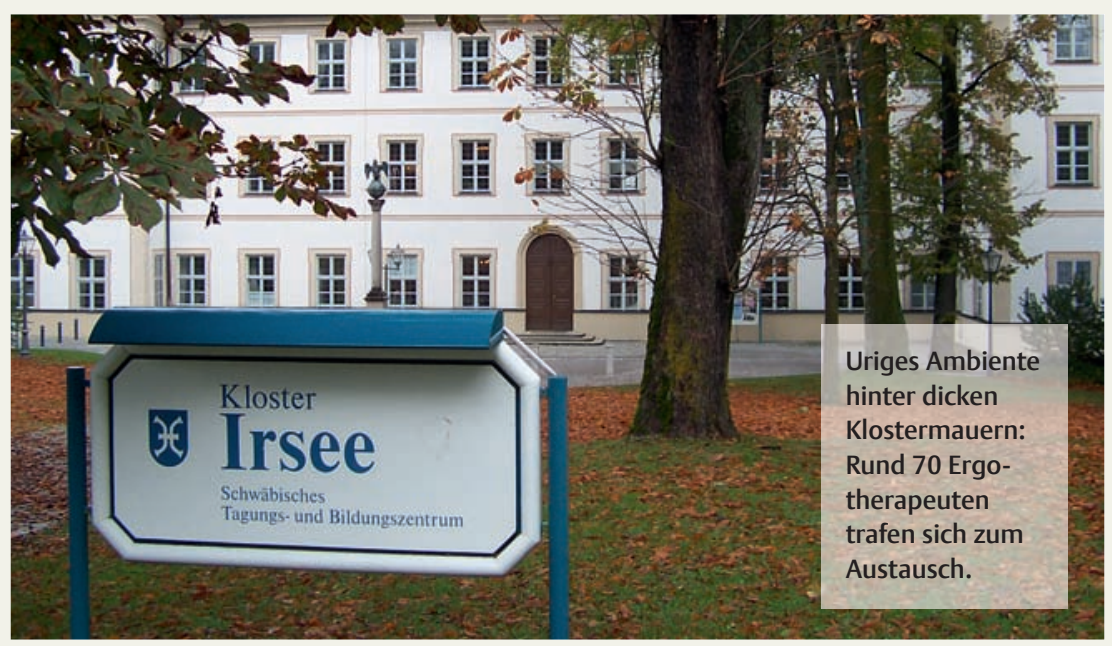

Zum dritten Mal in Folge konnte der Dreiländerkongress im bayerischen Irsee psychiatrisch tätige Ergotherapeuten von sich überzeugen: Rund 70 Therapeuten aus Deutschland, Österreich und der Schweiz nahmen vom 23. bis 25. Oktober 2011 am größten deutschsprachigen Kongress zur Ergotherapie in der Psychiatrie teil.

Die Therapeuten erfuhren in Vorträgen, Workshops und Konzeptbörsen auf einen Schlag mehr über aktuelle psychiatrische Entwicklungen. So konnten sich die Teilnehmer aus vier ganztägigen Workshops zu bestimmten Themen einen Workshop aussuchen. Sie bekamen Anregungen für Fortbildungen und konnten sich mit Kollegen aus anderen Einrichtungen austauschen, die meist mit ähnlichen Schwierigkeiten zu kämpfen haben. Die Referenten waren fast ausschließlich praktizierende Ergotherapeuten, die in ihrer Einrichtung das umsetzen, was sie auf dem Kongress vorstellten.

Zum Beispiel berichtete Marie Hofmann aus Deutschland, dass sie und ihre Kolleginnen das Handwerk aus der Rehaklinik für suchtkranke Menschen verbannt haben. Birgit Elsayed-Glaser aus Österreich zeigte anhand von Beispielen aus ihrer ambulanten Praxis, wie sie mit psychisch kranken Menschen den Alltag gestaltet. Theresa Witschi aus der Schweiz widmete sich dem Thema Depression im Rahmen der stationären Ergotherapie und diskutierte mit den Teilnehmern, wie man angemessen auf das Leiden der Betroffenen eingehen könne.

Eine Besonderheit im Jahr 2011 waren die Beiträge von psychiatrieerfahrenen Gästen. Zum Beispiel führte der Schweizer Filmemacher Dieter Gränicher seinen Film „Seelenschatten“ vor. Der Film begleitet über anderthalb Jahre drei Menschen, die an Depressionen leiden. Auf intensive und einfühlsame Weise machte Gränicher, der selbst auch Depressionen hatte, deutlich, wie Betroffene ihre Erkrankung erleben.

Der nächste Dreiländerkongress findet vom 21. bis 23. Oktober 2012 statt. Wichtige Informationen dazu findet man bereits jetzt unter www.bildungswerk-irsee.de > „Programm 2012“ > „Psychiatrie“.

czy
WEITERBILDUNGSSTUDIENGANG

\section{Fit für die Garten- therapie}

- Die Zürcher Hochschule für Angewandte Wissenschaften (ZHAW) bietet ab März 2012 den berufsbegleitenden Weiterbildungsstudiengang Gartentherapie an. Ergotherapeuten, die sich für die Beziehung Mensch Pflanze interessieren, finden auf der Internetseite www.iunr.zhaw.ch/gartentherapie alle wichtigen Inhalte zu dem Zertifikatslehrgang. Das Studium befähigt die Teilnehmer, gartentherapeutische Interventionen durchzuführen und Gärten für therapeutische Zwecke zu planen. Nach 12 Monaten und erfolgreicher Abschlussarbeit erhalten die Teilnehmer das "Certificate of Advanced Studies (CAS) in Gartentherapie“. Die Kosten dafür betragen 7.400 Schweizer Franken. Anmeldeschluss ist der 6. Februar 2012.

czy

TERMINE

Kongress für Gesundheitsberufe: Klinische Expertise sprengt Grenzen

2.-3. März 2012, Bern (Schweiz) www.insel.ch/klin_exp.html

2. Wittener Tagung zur Hilfsmittelversorgung: „Vielfalt oder Dschungel - der Versorgungsprozess mit Hilfsmitteln“" 9. März 2012, Witten www.hilfsmitteltagung.de

Münchner Symposium Frühförderung - „Frühförderung: Teilhabe inclusive?"“ 9.-10. März 2012, München www.fruehfoerderung-bayern.de > „Veranstaltungen“

$\Rightarrow$ Weitere Termine finden Sie unter www.thieme.de/ergoonline $>$ „Termine“. 


\section{Profession \& Perspektiven}

\section{WER WAR EIGENTLICH AUGUSTA DÉJERINE-KLUMPKE?}

\section{Eine starke Frau, die den Männern Paroli bot}

-Meine Mutter lehrte uns, mit Willenskraft und Beharrlichkeit durchs Leben zu gehen und bei Schwierigkeiten nicht aufzugeben." Das ist sicher ein Grund, warum Augusta Marie Déjerine-Klumpke (18591927) eine steile Karriere hinlegte und ihr Name in die medizinische Terminologie einging. Sie ist vor allem durch die „KlumpkeLähmung“ bekannt, einer Spinalnervenläsion des unteren Plexus brachialis.

Früh lernten Augusta und ihre fünf Geschwister, Neuem gegenüber aufgeschlossen zu sein und den eigenen Weg unbeirrt zu gehen. 1871 zog die Mutter mit ihnen von San Francisco nach Deutschland und weiter in die Schweiz, wo Augusta in der Oberschule für Mädchen in Lausanne schnell auffiel: Sie wurde Präsidentin des Schulmädchenvereins, ging mit ihrer Schwester ohne Anstandsdame zum Tanzen und rauchte in der Öffentlichkeit Zigaretten. Die Krönung des Ganzen: Augusta wollte Medizin studieren - und das als Frau!

In Lausanne gab es keine medizinische Fakultät, deswegen zog die Mutter kurzerhand mit ihren Kindern nach Paris. 1882 nahm Augusta ihr Medizinstudium auf gegen den Willen des Dekans. Sie war eine ehrgeizige, fleißige Studentin, die stets mit
Bestnoten abschloss. Mithilfe des Bildungsministers, der die Frauenbewegung unterstützte, wurde Augusta 1887 trotz massiver Widerstände der männlichen Klinikleitung als erste Frau am L'Hôpital de Lourcine „Interne des Hôpitaux“, was etwa dem deutschen Assistenzarzt entspricht.

Augustas herausragende Fähigkeiten waren einem Mann bereits während ihres Studiums aufgefallen: ihrem Lehrer, dem Neurologen und Chefarzt, Joseph Jules Déjerine. Im Jahr 1888 heirateten die beiden, was der Beginn einer einzigartigen Zusammenarbeit war. Das Ehepaar forschte gemeinsam und veröffentlichte unter anderem das in zwei Bänden erschienene Werk „Anatomie des centres nerveux“. Es wurde zum Standardwerk in der Neurologie. Im Jahr 1889 promovierte Augusta mit der Arbeit über Polyneuritis und die Auswirkungen von Bleivergiftungen auf das Nervensystem. Viele waren im 19. Jahrhundert von Bleivergiftungen betroffen, denn zum Verlöten von Konservendosen wurden damals Bleilegierungen verwendet.

Allein zwischen 1885 und 1914 veröffentlichte Augusta insgesamt 56 bedeutsame wissenschaftliche Arbeiten unter ihrem Namen. Ihre Beiträge zu den Publika- tionen ihres Mannes blieben hingegen häufig ungenannt. Das schmälerte den Zusammenhalt des Ehepaars allerdings keineswegs. So sprang Augusta beispielsweise auf einer Tagung der Neurologischen Gesellschaft von Paris für ihren Mann ein und bot dessen Kontrahenten mit ihrem klinisch-anatomischen Wissen Paroli. Diese Auseinandersetzung ging als „Aphasie-Streit“ in die Geschichte ein. Ein paar Jahre später krönte Augusta ihre Karriere, indem sie als erste Frau zur Präsidentin dieser Neurologischen Gesellschaft gewählt wurde.

Bemerkenswerte Laufbahnen legten auch ihre Geschwister hin: Anna Elisabeth wurde eine bekannte amerikanische Malerin, Dorothee Astronomin, Mathilda eine Pianistin, Julia Violonistin und John William Ingenieur. Am Ende ihres Lebens resümierte Augusta Déjerine-Klumpke: „Mir scheint so, dass meiner geliebten Mutter die Förderung ihrer Kinder nicht allzu schlecht geglückt ist." Man kann ihr nur beipflichten.

giro

\section{UNTERSUCHUNG}

\section{Singen macht schlau}

Kinder, die früh singen, werden schneller schulreif als Kinder, die nicht singen. Das fanden Forscher der Universität Bielefeld heraus. Ein Team um den Soziologen Thomas Blank untersuchte bei 500 Fünfjährigen, inwiefern häufiges Singen mit deren Schulreife zusammenhängt. Uneingeweihte Ärzte schätzten 88 Prozent der „Sänger“ als regelschulreif ein, aber nur 44 Prozent der „Nicht-Sänger“. Den positiven Effekt von Musik auf intellektuelle und soziale Fähigkeiten scheint diese Untersuchung zu bestätigen.

czy 


\section{ЈОВ-О-ЈОВ}

\section{Neues Jobportal}

Seit 2011 ist eine neue Internetseite auf dem Markt mit dem Schwerpunkt Jobvermittlung für Therapeuten. Die Seite www.job-o-job.de bietet neben den Stellenangeboten auch weitere Funktionen an. Zum Beispiel hilft eine Zimmerbörse bei der Suche nach günstigen Übernachtungsmöglichkeiten.

Noch ist das Portal am Entstehen. Das zeigen die gerade mal drei Stellenangebote. Sollte es sich etablieren können, wäre es sicher ein Gewinn für alle arbeitssuchenden Therapeuten.

\section{HÄNDEDESINFEKTION}

\section{So geht's richtig}

Ergotherapeuten, die ihre Hände häufig waschen und desinfizieren müssen, können schnell Juckreiz, Trockenheit oder Ausschläge bekommen. Es sind aber nicht die alkoholhaltigen Desinfektionsmittel, die der Haut Schaden zufügen. Verursacher allen Übels sind die detergenzienhaltigen Handwaschmittel. Der Wasserverlust der Haut ist nach dem Händewaschen höher als nach der Desinfektion. Das Positive am Desinfizieren ist, dass der Alkohol die überschüssigen Detergenzien des Waschmittels entfernt und so das Austrocknen der Haut vermindert. Ein Tipp für die Praxis: die Hände konsequent desinfizieren, das Händewaschen ist nur bei sichtbaren Verschmutzungen nötig. Je einfacher und verträglicher die Handhygiene, desto konsequenter führt man sie schließlich durch.

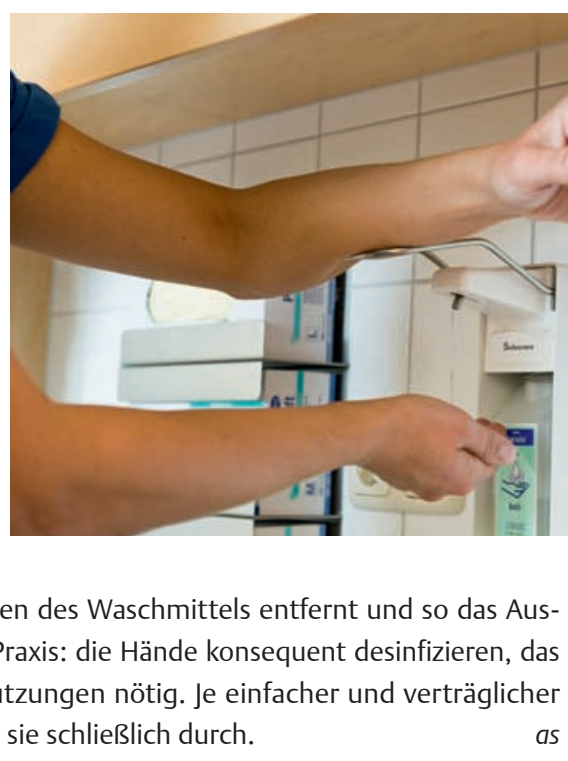

\section{VERANSTALTUNG}

\section{Promovierende unterstützen}

Die Promotion ist für Therapeuten ein noch recht neuer Weg, der aber immer mehr gewählt wird. Orientierung bot die Veranstaltung „Empowerment für die Promotion in den Gesundheitsberufen“, die am 13. Oktober 2011 in Halle stattfand. Etwa 80 Teilnehmer folgten der Einladung des Hochschulverbundes für Gesundheitsfachberufe (HVG) und der Internationalen Graduiertenakademie der Universität Halle-Wittenberg. Ziel war es, junge Nachwuchswissenschaftler darunter auch Ergotherapeuten - zu stärken und sie in ihrer Promotion zu unterstützen.
Außerdem wollten die Veranstalter herausfinden, welche spezifischen Bedürfnisse der wissenschaftliche Nachwuchs in den Gesundheitsfachberufen hat.

Spannend war das Konzept des sogenannten Weltcafés: In kleiner Runde trafen sich Experten und Interessierte an insgesamt sechs Thementischen rund um die Promotion. Dort konnten sie ihr Wissen und ihre Erfahrungen austauschen, Ideen besprechen und Lösungen für Probleme entwickeln. Das Angebot der Thementische bot für jeden etwas, beispielsweise „Promovieren - aber wo? Promovieren in der eigenen Disziplin oder in Bezugswissenschaften?“, „Das Ganze auf die Füße stellen! Finanzielle Förderungsmöglichkeiten“ und „Tue Gutes und schreibe darüber! Treffen mit Vertretern der Fachverlage“. Be- liebt war auch der Tisch zum Thema „Ein Blick über den Tellerrand! Promovieren im Ausland“. Die Teilnehmer wechselten nach Bedarf die einzelnen Tische. So entstand eine dynamische und konstruktive Diskussion, nicht nur mit den Experten, sondern auch unter den Teilnehmern.

Es besteht derzeit ein großer Bedarf an hochqualifizierten Therapeuten. Zum einen, weil sie Studiengänge mitaufbauen, zum anderen, weil sie an Hochschulen unterrichten. Das zeigte auch die Nachfrage an der Veranstaltung: Die Therapeuten wünschen sich umfassende Informationen zur wissenschaftlichen Nachwuchsförderung. Der HVG plant daher weitere Veranstaltungen.

Rachel Sommer, Bachelor of Health in OT (NL), Master of Public Health 